Supporting Information of

\title{
Relative importance of gas uptake on aerosol and ground surfaces characterized by equivalent uptake coefficients
}

\author{
Meng $\mathrm{Li}^{1}$, Hang $\mathrm{Su}^{2,1}$, Guo $\mathrm{Li}^{1}$, Nan $\mathrm{Ma}^{2}$, Ulrich Pöschl ${ }^{1}$, and Yafang Cheng ${ }^{1}$ \\ ${ }^{1}$ Max Planck Institute for Chemistry, Mainz, 55118, Germany \\ ${ }^{2}$ Center for Air Pollution and Climate Change Research (APCC), Institute for Environmental and \\ Climate Research (ECI), Jinan University, Guangzhou, 511443, China
}

Correspondence to: Hang Su (h.su@mpic.de) and Yafang Cheng (yafang.cheng@mpic.de) 


\section{Contents}

Calculation of dry deposition velocities above the ground surface for the investigated gases.

\section{Tables}

Table S1. Friction velocity and canopy roughness length by land use type.

Table S2. Seasonal mean dry deposition velocities by gas species, unit: $\mathrm{cm} \mathrm{s}^{-1}$.

Table S3. Seasonal $\gamma_{\text {eqv }}$ by gas species at typical condition (typical aerosol area density of $A$ as described in the main text, and mixing height of $300 \mathrm{~m}), \times 10^{-4}$.

\section{Figures}

Figure S1. Resistance model for dry deposition, derived from the Figure 19.1 in Seinfeld and Pandis (2006).

Figure S2. Schematic model for canopy resistance, derived from Wesely (1989). 
Calculation of dry deposition velocities above the ground surface for gases

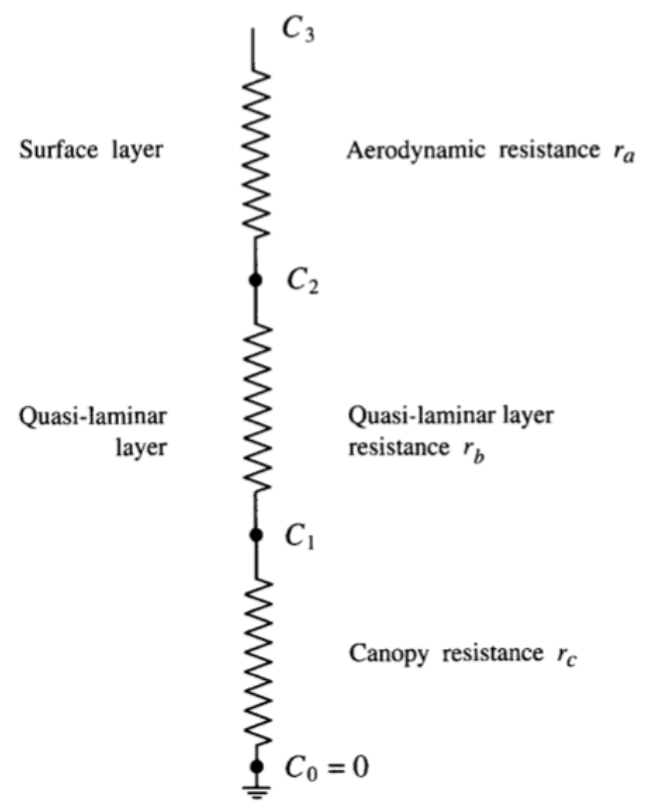

Figure S1. Resistance model for dry deposition, derived from the Figure 19.1 in Seinfeld and Pandis (2006).

$V_{d}=\frac{1}{R_{a}+R_{b}+R_{c}}$

Following Wesely (1989) and Zhang et al. (2003), we calculated $\mathrm{R}_{\mathrm{a}}$ (aerodynamic resistance), $\mathrm{R}_{\mathrm{b}}$ (quasi-laminar layer resistance) and $\mathrm{R}_{\mathrm{c}}$ (canopy resistance) as below.

\section{1 calculation of $R_{\mathrm{a}}$}

Under neutral atmospheric condition:

$R_{a}=\int_{Z_{0}}^{Z} \frac{1}{\kappa u_{*} z} d z=\frac{1}{\kappa u_{*}} \ln \left(\frac{Z}{Z_{0}}\right)$

where $\kappa$ is the von Karman constant (about 0.41 ); $\mathrm{u} *$ means the friction velocity (in unit of $\mathrm{m} \mathrm{s}^{-1}$ ); $\mathrm{Z}_{0}$ is the roughness length (in unit of $\mathrm{m}$ ); $\mathrm{Z}$ is the PBL mixing height (in unit of $\mathrm{m}$ ), we use a typical value of $\mathrm{Z}$ as $300 \mathrm{~m}$.

For different land use type, we assign different $\mathrm{u} *$ following the parameterization scheme of Zhang et al. (2003), and $Z_{0}$ based on Seinfeld and Pandis (2006): 
Table S1. Friction velocity and canopy roughness length by land use type.

\begin{tabular}{|l|l|l|l|l|}
\hline $\begin{array}{l}\text { land use } \\
\text { index }\end{array}$ & land use type & $\mathrm{u} *$ day $\left(\mathrm{m} \mathrm{s}^{-1}\right)$ & $\mathrm{u} *$ night $\left(\mathrm{m} \mathrm{s}^{-1}\right)$ & $\mathrm{Z}_{0}(\mathrm{~m})$ \\
\hline 1 & urban land & 0.6 & 0.3 & 1 \\
\hline 2 & agricultural land & 0.4 & 0.2 & 0.1 \\
\hline 3 & range land & 0.4 & 0.2 & 0.1 \\
\hline 4 & deciduous forest & 0.6 & 0.3 & 1 \\
\hline 5 & coniferous forest & 0.6 & 0.3 & 0.9 \\
\hline 6 & $\begin{array}{l}\text { mixed forest including } \\
\text { wetland }\end{array}$ & 0.6 & 0.3 & 0.9 \\
\hline 7 & water & 0.3 & 0.25 & 0.1 \\
\hline 8 & barren land, desert & 0.25 & 0.15 & 0.04 \\
\hline 9 & non-forested wetland & 0.25 & 0.2 & 0.1 \\
\hline 10 & $\begin{array}{l}\text { mixed agricultural and } \\
\text { range land }\end{array}$ & 0.4 & 0.2 & 0.1 \\
\hline 11 & $\begin{array}{l}\text { rocky open areas with } \\
\text { low-growing shrubs }\end{array}$ & 0.4 & 0.6 & 0.3 \\
\hline
\end{tabular}

\section{2 calculation of $R_{b}$}

$R_{b}=\frac{5 S c^{2 / 3}}{u_{*}} \quad S c=\frac{v}{D}$

where Sc is the Schmidt number (unitless), v means the kinematic viscosity of air, D is the molecular diffusivity for gases. We use $\mathrm{D}=10^{-5} \mathrm{~m}^{2} \mathrm{~s}^{-1}$, and a temperature-dependent $\mathrm{v}$ in the calculation.

\section{3 calculation of $R_{c}$}




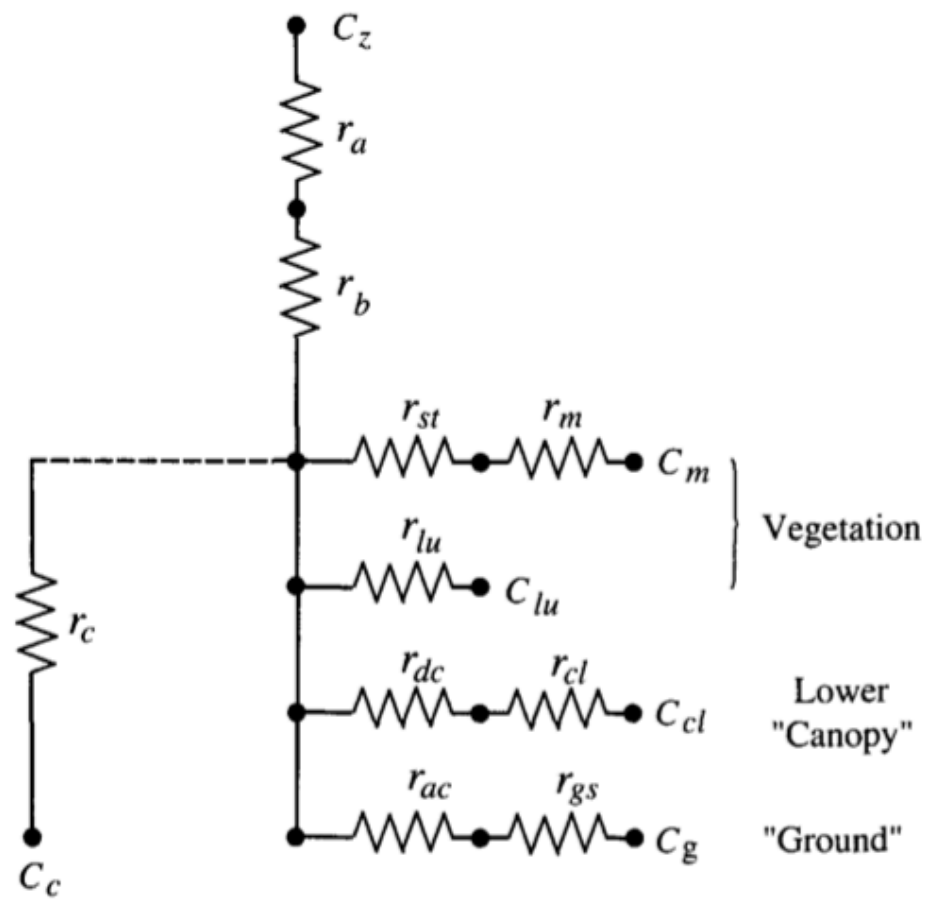

Figure S2. Schematic model for canopy resistance, derived from Wesely (1989).

$R_{c}=\left(\frac{1}{R_{s t}+R_{m}}+\frac{1}{R_{l u}}+\frac{1}{R_{d c}+R_{c l}}+\frac{1}{R_{a c}+R_{g s}}\right)^{-1}$

As illustrated by Seinfeld and Pandis (2006), $\mathrm{R}_{\text {st }}$ represents the resistance of the leaf stomatal, $\mathrm{R}_{\mathrm{m}}$ for mesophyll resistance, $\mathrm{R}_{\mathrm{lu}}$ is the surface resistance in the upper canopy, $\mathrm{R}_{\mathrm{dc}}$ means the resistance by buoyant convection, $\mathrm{R}_{\mathrm{cl}}$ is the uptake resistance by leaves, twigs and etc., $\mathrm{R}_{\mathrm{ac}}$ means the transfer resistance for processes at the ground, and $\mathrm{R}_{\mathrm{gs}}$ is the uptake resistance by soil, leaf litter and others on the ground surface.

The equations to calculate each item of $R_{c}$ are illustrated in Wesely (1989). The important input parameters for Rc calculation include: the input resistance by land use and season, the physical and chemical reactivity scales by gas species, and the meteorological parameters. We adopted the the parameterization scheme of Wesely (1989) for the former two items of input, and a set of typical hourly temperature and radiation values for each season derived from the standard meteorological database for construction in China (Zhang, 2004).

The calculated dry deposition velocities by gas and land use type for each season are presented in Table S2. Furthermore, we show the seasonal equivalent uptake coefficients $\left(\gamma_{\mathrm{eq}}\right)$ at typical conditions based on the dry deposition velocities in Table S3. 
Table S2. Seasonal mean dry deposition velocities by gas species, unit: $\mathrm{cm} \mathrm{s}^{-1}$.

\begin{tabular}{|c|c|c|c|c|c|}
\hline Gases & Winter & Spring & Summer & $\begin{array}{l}\text { Autumn with unharvested } \\
\text { cropland }\end{array}$ & $\begin{array}{c}\text { Late autumn after } \\
\text { frost }\end{array}$ \\
\hline \multicolumn{6}{|c|}{ Urban } \\
\hline $\mathrm{O}_{3}$ & 0.15 & 0.24 & 0.24 & 0.24 & 0.24 \\
\hline $\mathrm{NO}_{2}$ & 0.02 & 0.04 & 0.04 & 0.04 & 0.04 \\
\hline $\mathrm{SO}_{2}$ & 0.41 & 0.17 & 0.20 & 0.20 & 0.20 \\
\hline $\mathrm{N}_{2} \mathrm{O}_{5}$ & 2.10 & 2.26 & 2.31 & 2.14 & 2.14 \\
\hline $\mathrm{HNO}_{3}$ & 2.10 & 2.26 & 2.31 & 2.14 & 2.14 \\
\hline $\mathrm{H}_{2} \mathrm{O}_{2}$ & 0.44 & 0.32 & 0.34 & 0.33 & 0.33 \\
\hline \multicolumn{6}{|c|}{ Agricultural land } \\
\hline $\mathrm{O}_{3}$ & 0.07 & 0.45 & 0.44 & 0.29 & 0.41 \\
\hline $\mathrm{NO}_{2}$ & 0.02 & 0.20 & 0.29 & 0.07 & 0.07 \\
\hline $\mathrm{SO}_{2}$ & 0.49 & 0.43 & 0.42 & 0.24 & 0.40 \\
\hline $\mathrm{N}_{2} \mathrm{O}_{5}$ & 1.10 & 1.18 & 1.21 & 1.12 & 1.12 \\
\hline $\mathrm{HNO}_{3}$ & 1.10 & 1.18 & 1.21 & 1.12 & 1.12 \\
\hline $\mathrm{H}_{2} \mathrm{O}_{2}$ & 0.51 & 0.57 & 0.51 & 0.34 & 0.57 \\
\hline \multicolumn{6}{|c|}{ Amazon forest } \\
\hline $\mathrm{O}_{3}$ & 0.15 & 0.27 & 0.37 & 0.14 & 0.14 \\
\hline $\mathrm{NO}_{2}$ & 0.04 & 0.17 & 0.28 & 0.04 & 0.04 \\
\hline $\mathrm{SO}_{2}$ & 0.14 & 0.25 & 0.34 & 0.14 & 0.14 \\
\hline $\mathrm{N}_{2} \mathrm{O}_{5}$ & 2.10 & 2.26 & 2.31 & 2.14 & 2.14 \\
\hline $\mathrm{HNO}_{3}$ & 2.10 & 2.26 & 2.31 & 2.14 & 2.14 \\
\hline $\mathrm{H}_{2} \mathrm{O}_{2}$ & 0.23 & 0.37 & 0.48 & 0.23 & 0.23 \\
\hline \multicolumn{6}{|c|}{ Water } \\
\hline $\mathrm{O}_{3}$ & 0.07 & 0.07 & 0.07 & 0.06 & 0.06 \\
\hline $\mathrm{NO}_{2}$ & 0.01 & 0.01 & 0.01 & 0.01 & 0.01 \\
\hline $\mathrm{SO}_{2}$ & 0.03 & 0.03 & 0.03 & 0.03 & 0.03 \\
\hline $\mathrm{N}_{2} \mathrm{O}_{5}$ & 1.05 & 1.06 & 1.07 & 1.05 & 1.05 \\
\hline $\mathrm{HNO}_{3}$ & 1.05 & 1.06 & 1.07 & 1.05 & 1.05 \\
\hline $\mathrm{H}_{2} \mathrm{O}_{2}$ & 0.08 & 0.08 & 0.08 & 0.08 & 0.08 \\
\hline
\end{tabular}


Table S3. Seasonal $\gamma_{\text {eqv }}$ by gas species at typical condition (typical aerosol area density of $A$ as described in the main text, and mixing height of $300 \mathrm{~m}), \times 10^{-4}$.

\begin{tabular}{|c|c|c|c|c|c|}
\hline Gases & Winter & Spring & Summer & $\begin{array}{l}\text { Autumn with unharvested } \\
\text { cropland }\end{array}$ & $\begin{array}{c}\text { Late autumn after } \\
\text { frost }\end{array}$ \\
\hline \multicolumn{6}{|c|}{ Urban } \\
\hline $\mathrm{O}_{3}$ & 0.64 & 1.01 & 1.01 & 1.00 & 1.00 \\
\hline $\mathrm{NO}_{2}$ & 0.10 & 0.15 & 0.15 & 0.15 & 0.15 \\
\hline $\mathrm{SO}_{2}$ & 1.73 & 0.72 & 0.84 & 0.83 & 0.83 \\
\hline $\mathrm{N}_{2} \mathrm{O}_{5}$ & 8.91 & 9.59 & 9.78 & 9.07 & 9.07 \\
\hline $\mathrm{HNO}_{3}$ & 8.91 & 9.59 & 9.78 & 9.07 & 9.07 \\
\hline $\mathrm{H}_{2} \mathrm{O}_{2}$ & 1.87 & 1.37 & 1.44 & 1.41 & 1.41 \\
\hline \multicolumn{6}{|c|}{ Agricultural land } \\
\hline $\mathrm{O}_{3}$ & 1.31 & 8.76 & 8.55 & 5.63 & 7.93 \\
\hline $\mathrm{NO}_{2}$ & 0.30 & 3.88 & 5.57 & 1.38 & 1.36 \\
\hline $\mathrm{SO}_{2}$ & 9.45 & 8.39 & 8.12 & 4.55 & 7.69 \\
\hline $\mathrm{N}_{2} \mathrm{O}_{5}$ & 21.21 & 22.88 & 23.37 & 21.65 & 21.65 \\
\hline $\mathrm{HNO}_{3}$ & 21.21 & 22.88 & 23.37 & 21.65 & 21.65 \\
\hline $\mathrm{H}_{2} \mathrm{O}_{2}$ & 9.80 & 10.97 & 9.94 & 6.61 & 11.06 \\
\hline \multicolumn{6}{|c|}{ Amazon forest } \\
\hline $\mathrm{O}_{3}$ & 14.01 & 25.72 & 35.56 & 13.78 & 13.78 \\
\hline $\mathrm{NO}_{2}$ & 3.88 & 16.54 & 27.18 & 3.56 & 3.56 \\
\hline $\mathrm{SO}_{2}$ & 13.96 & 24.22 & 33.03 & 13.76 & 13.76 \\
\hline $\mathrm{N}_{2} \mathrm{O}_{5}$ & 203.33 & 218.79 & 223.13 & 207.08 & 207.08 \\
\hline $\mathrm{HNO}_{3}$ & 203.33 & 218.79 & 223.13 & 207.08 & 207.08 \\
\hline $\mathrm{H}_{2} \mathrm{O}_{2}$ & 22.43 & 35.42 & 45.95 & 22.25 & 22.25 \\
\hline \multicolumn{6}{|c|}{ Water } \\
\hline $\mathrm{O}_{3}$ & 3.92 & 3.81 & 3.82 & 3.76 & 3.76 \\
\hline $\mathrm{NO}_{2}$ & 0.70 & 0.53 & 0.53 & 0.49 & 0.49 \\
\hline $\mathrm{SO}_{2}$ & 1.76 & 1.66 & 1.67 & 1.61 & 1.61 \\
\hline $\mathrm{N}_{2} \mathrm{O}_{5}$ & 61.37 & 62.24 & 62.36 & 61.33 & 61.33 \\
\hline $\mathrm{HNO}_{3}$ & 61.37 & 62.24 & 62.36 & 61.33 & 61.33 \\
\hline $\mathrm{H}_{2} \mathrm{O}_{2}$ & 4.68 & 4.61 & 4.63 & 4.51 & 4.51 \\
\hline
\end{tabular}




\section{Reference}

Seinfeld, J. H., and Pandis, S. N.: Atmosppheric chemistry and physics: From air pollution to climate change, John Wiley \& Sons, New York, USA, 2006.

Wesely, M. L.: Parameterization of surface resistances to gaseous dry deposition in regional-scale numerical models, Atmospheric Environment (1967), 23, 1293-1304, https://doi.org/10.1016/0004-6981(89)90153-4, 1989.

Zhang, L., Brook, J. R., and Vet, R.: A revised parameterization for gaseous dry deposition in air-quality models, Atmos. Chem. Phys., 3, 2067-2082, 10.5194/acp-32067-2003, 2003.

Zhang, Q.: China Standard Meteorological Database for Construction (CD-ROM), China Mechanical Industry Press, Beijing, 2004. 